Contact Dr Zoe Webster.

E-mail: zoe.webster@ccdhb.org.nz

1. Hannibal L, Lysne V, Bjørke-Monsen, et al. Biomarkers and algorithms for the diagnosis of vitamin B 12 deficiency. Front Mol Biosci 2016; 3: 27.

2. Calderon-Ospina C, Nava-Mesa M. B vitamins in the nervous system: current knowledge of the biochemical modes of action and synergies of thiamine, pyridoxine, and cobalamin. CNS Neurosci Ther 2020; 26 : $5-13$

3. Kumar N. Neurologic aspects of cobalamin (B12) deficiency. Handb Clin Neurol 2014; 120: 915-26.

4. McCaddon A. Vitamin B12 in neurology and ageing; clinical and genetic aspects. Biochimie 2013; 95: 1066-76.

5. Ontario Health Technology Advisory Committee (OHTAC). Testing Vitamin B12 Levels in Neuropathy, Alopecia, Dizziness, and Fatigue: OHTAC Recommendation. Toronto: Health Quality Ontario, 2014 https://www.hqontario.ca/Portals/0/Documents/evidence/reports/ recommendation-testing-vitamin-b12-levels-140203-en.pdf

6. Saperstein DS, Wolfe GI, Gronseth GS, et al. Challenges in the identification of cobalamin-deficiency polyneuropathy. Arch Neurol 2003 60: 1296-301.

7. Overell JR. Peripheral neuropathy: pattern recognition for the pragmatist. Pract Neurol 2011; 11: 62-70.

8. Moore E, Mander A, Ames D, et al. Cognitive impairment and vitamin B12: a review Int Psychogeriatr 2012; 24: 541-56.

9. Lachner C, Steinle NI, Regenold WT, et al. The neuropsychiatry of vitamin B12 deficiency in elderly patients. J Neuropsychiatr Clin Neurosci 2012; 24: 5-15.

10. Wolffenbuttel BHR, Wouters HJCM, Heiner-Fokkema MR, et al. The many faces of cobalamin (vitamin $\mathrm{B}_{12}$ ) deficiency. Mayo Clin Proc Innov Qual Outcome 2019; 3: 200-14.

11. 3D (Lower North Island) Community Health Pathways. B12 deficiency. Apr 2019; cited Oct 2020. https://3d.communityhealthpathways.org/ 16981.htm.

DOI: https://doi.org/10.1016/j.pathol.2021.02.008

\section{L-asparaginase-induced biochemical toxicities in young adults with acute lymphoblastic leukaemia and T-lymphoblastic lymphoma}

Sir,

For over 50 years, extracellular depletion of asparagine using L-asparaginase has been used successfully as a chemotherapeutic treatment for haematological malignancies. ${ }^{1}$ More recently, asparagine has been shown to play a critical role in regulating cellular adaptation to glutamine depletion and that glutaminolysis induces apoptosis. ${ }^{2}$ First trialled in a child with acute lymphoblastic leukaemia (ALL) in 1966, L-asparaginase produced a marked reduction in circulating lymphoblasts, but was associated with severe haemolysis and fatal pulmonary haemorrhage. ${ }^{3}$ Its use has substantially reduced the mortality due to ALL. However, its short therapeutic plasma half-life and toxic side effects including hypersensitivity reactions, coagulation disturbances and anaphylaxis, remain ongoing challenges for treating physicians. ${ }^{1}$ In the short-acting or pegylated forms, L-asparaginase is a backbone of current paediatric protocols for ALL, but has only recently been added to young adult protocols due to the excess of treatmentrelated toxicities observed in older when compared with younger patients. ${ }^{4}$

We report a case series demonstrating some of the biochemical toxicities experienced by four young adults receiving L-asparaginase as part of their treatment protocols at our institution and discuss the need to monitor for these complications in this patient group.

Case 1 was an 18-year-old male with no prior history of diabetes. He presented to the emergency department unwell and obtunded during salvage therapy for his second relapse of T-ALL, requiring admission to the intensive care unit. He was febrile, neutropenic, in supraventricular tachycardia, hyponatraemic $(\mathrm{Na} 127 \mathrm{mmol} / \mathrm{L})$ and in severe diabetic ketoacidosis (DKA) $\left(\mathrm{pH} 6.99, \mathrm{HCO}_{3}-4.0 \mathrm{mmol} / \mathrm{L}\right.$, anion gap 34 $\mathrm{mmol} / \mathrm{L}$, glucose $48.7 \mathrm{mmol} / \mathrm{L}$ ) (Table 1). His initial therapy (HyperCVAD, POMP) and treatment for first relapse (BFM: phase I and phase II consolidation, maintenance) had not included L-asparaginase, as this was prior to its inclusion in standard young adult protocols. A suitable stem cell donor was not identified. His treatment at second relapse included re-induction with the BFM protocol with the addition of pegL-asparaginase 3750 units IM on day 1 and dexamethasone 16 mg orally for 21 days, leading to the presentation with DKA. He recovered following appropriate management and required long-term insulin therapy. At review, he reported a preceding one month history of polydipsia and polyuria. His C-peptide level was undetectable and islet antibody testing was negative. A diagnosis of de novo type 1 diabetes mellitus, unmasked by chemotherapy and sepsis was made. Treatment with L-asparaginase was abandoned.

Case 2 was a 17 -year-old female with T-ALL who was treated with L-asparaginase and long-term corticosteroids as part of the Children's Cancer Group (CCG) AALL0232 study protocol. The intensification and consolidation phases were complicated by the development of type 2 diabetes and marked hyperlipidaemia. A random blood glucose level of 30 $\mathrm{mmol} / \mathrm{L}$ was detected upon a presentation to the emergency department with polyuria, polydipsia and fatigue (Table 1). There was no associated ketonuria and her C-peptide level was normal. Glutamic acid decarboxylase (GAD) and islet cell antibody testing was negative. She required subcutaneous insulin therapy that was able to be ceased following completion of the steroids and L-asparaginase. The hyperlipidaemia became apparent due to subsequent markedly aberrant biochemical blood test results, including hyponatraemia, which proved discordant with a concurrently run

Table 1 Laboratory biochemical and molecular findings

\begin{tabular}{|c|c|c|c|c|c|}
\hline & \multicolumn{4}{|c|}{ Case } & \multirow{2}{*}{$\begin{array}{c}\text { Reference } \\
\text { interval/limit }\end{array}$} \\
\hline & 1 & 2 & 3 & 4 & \\
\hline Gender (M/F) & M & $\mathrm{F}$ & M & M & \\
\hline Age (years) & 18 & 17 & 17 & 17 & \\
\hline $\begin{array}{l}\text { Glucose } \\
\quad(\mathrm{mmol} / \mathrm{L})\end{array}$ & 48.7 & 30.0 & 14.4 & ND & $3.0-5.4$ \\
\hline Amylase (U/L) & 820 & 295 & 18 & 504 & $<100$ \\
\hline Lipase (U/L) & ND & 83 & 28 & 666 & $<40$ \\
\hline $\begin{array}{l}\text { Cholesterol } \\
(\mathrm{mmol} / \mathrm{L})\end{array}$ & ND & 14.6 & 17.5 & 3.0 & $<5.5$ \\
\hline $\begin{array}{l}\text { Triglyceride } \\
(\mathrm{mmol} / \mathrm{L})\end{array}$ & ND & 45.7 & 44.5 & 2.3 & $<1.8$ \\
\hline$A P O E$ genotype & ND & $\varepsilon 4 / 3$ & $\varepsilon 4 / 3$ & ND & \\
\hline$L P L$ & ND & No mutation & No mutation & ND & \\
\hline APOC 2 & ND & No mutation & No mutation & ND & \\
\hline APOA5 & ND & No mutation & $\begin{array}{l}\text { c. }-1131 \mathrm{~T}>\mathrm{C} \\
\text { heterozygote }\end{array}$ & ND & \\
\hline
\end{tabular}

F, female; M, male; ND, not done. 
venous blood gas sample. The sample was noted to be lipaemic on gross inspection, causing pseudo-hyponatraemia. A lipid profile revealed triglycerides of $45.7 \mathrm{mmol} / \mathrm{L}$ and total cholesterol $14.6 \mathrm{mmol} / \mathrm{L}$. The patient was contacted with a plan to start lipid-lowering therapy; however, given a history of several hours of abdominal pain, she was advised to attend the emergency department. She was subsequently diagnosed with acute pancreatitis [amylase $295 \mathrm{U} / \mathrm{L}(<100)$ ] that was attributed to the L-asparaginase-induced hyperlipidaemia. She recovered with conservative management and lipidlowering therapy including gemfibrozil, atorvastatin and fish oil capsules, which was ceased upon normalisation of her lipid profile following completion of the L-asparaginase. Subsequent doses of L-asparaginase were changed from the pegylated to the short-acting formulation, and her lipid profile and pancreatic enzymes were monitored closely. There were no further episodes of severe hyperlipidaemia or pancreatitis. Hypertriglyceridaemia genetic testing for mutations in $L P L$, $A P O A 5$ and $A P O C 2$ were negative.

Case 3 was a 17-year-old male with T-lymphoblastic lymphoma who was treated with the CCG AALL0232 protocol following initial induction with a cycle of HyperCVAD. The HyperCVAD with pre-phase dexamethasone was given while awaiting confirmatory diagnostic pathology due to an urgent need to commence therapy for thoracic outlet obstruction secondary to a mediastinal mass. He subsequently received a modified re-induction followed by standard therapy as per the CCG protocol. No complications occurred during the initial HyperCVAD and dexamethasone induction. Within one week of commencing the CCG protocol, which included first dosing of peg-L-asparaginase, he developed a coagulopathy [INR $1.0(0.9-1.3)$, APTT $61 \mathrm{~s}(27-36)$, fibrinogen 1.1 $\mathrm{g} / \mathrm{L}(2-4)]$ and moderate hyperglycaemia (glucose 14.4 $\mathrm{mmol} / \mathrm{L}$ ) (Table 1). The coagulopathy was consistent with $\mathrm{L}-$ asparaginase-induced dysfibrinogenaemia. He was treated with appropriate blood product replacement and monitoring whilst the hyperglycaemia was managed with a diabetic diet and monitoring. Lipid analyses during this time showed a triglyceride of $20.6 \mathrm{mmol} / \mathrm{L}$ (which had been normal at baseline) and total cholesterol of $6.4 \mathrm{mmol} / \mathrm{L}$. A further dose of peg-L-asparaginase was administered prior to commencement of any lipid-lowering strategies and subsequent blood samples were found to be grossly lipaemic causing laboratory analytical interference and revealed a triglyceride of 44.5 $\mathrm{mmol} / \mathrm{L}$ and total cholesterol of $17.5 \mathrm{mmol} / \mathrm{L}$. He presented concurrently with mild generalised abdominal pain, however, serum lipase and amylase were normal. He commenced lipidlowering therapy with gemfibrozil, atorvastatin and fish oil capsules to good effect. Hypertriglyceridaemia genetic testing revealed the APOA5 c.-1131C $>$ T variant (rs662799), which is associated with increased fasting triglyceride levels and increased risk of severe hypertriglyceridaemia.

Case 4 was a 17-year-old male with Philadelphia chromosome positive (Ph) ALL who was treated with the CCG protocol and imatinib. His treatment was supplemented with exogenous erythropoietin, folic acid and iron supplements to boost his bone marrow reserves as he was a Jehovah's Witness and had declined most therapeutic blood products. On one occasion the patient agreed to receive cryofibrinogen due to the development of L-asparaginase-induced dysfibrinogenaemia with associated severe thrombocytopenia. Within 4 days of his first dose of peg-L-asparaginase he presented with severe back pain, epigastric tenderness, fever and tachycardia. He was diagnosed with acute pancreatitis [lipase $666 \mathrm{U} / \mathrm{L}(<40)$, amylase $504 \mathrm{U} / \mathrm{L}$ $(<100)$ ] (Table 1), which resolved with conservative management. His lipids were not assessed at this time, but were normal at baseline.

The relatively recent addition of L-asparaginase to adolescent and young adult protocols for the treatment of ALL has shown promising effects on disease response in these patient groups. However, compliance in the setting of a prolonged treatment protocol and additional drug-related toxicities in young adults could prove problematic for adult haematologists outside of the trial setting. ${ }^{6}$ Experience in our local setting has revealed several biochemical toxicities attributable to L-asparaginase, occurring more frequently in this patient group than would be expected in a similarly treated paediatric population. Hyperlipidaemia, particularly severe hypertriglyceridaemia, with or without acute pancreatitis, has been a recurring theme in our young adult patients treated with the drug. Although hyperlipidaemia is relatively common in paediatric patients receiving L-asparaginase, severe hypertriglyceridaemia with associated acute pancreatitis is not. ${ }^{7-9}$

The lipid abnormalities seen during L-asparaginase-containing chemotherapy for ALL in paediatric populations have been reported to be benign, transient and self-remitting. However, the rare development of severe hypertriglyceridaemia $(>11.3$ $\mathrm{mmol} / \mathrm{L}$ ) in paediatric patients is considered high risk for the development of acute pancreatitis and intervention and frequent monitoring has been recommended once this threshold is reached. ${ }^{8,9}$ L-asparaginase-related acute pancreatitis has been reported to also occur in the paediatric population in the absence of associated hypertriglyceridaemia. ${ }^{9}$ In one study where 7 of 31 asparaginase-treated children with ALL were diagnosed with asparaginase-associated pancreatitis, there was no difference in triglyceride levels in patients diagnosed with pancreatitis compared to those who were not. ${ }^{10}$ The other rare clinical complication of severe hypertriglyceridaemia that has been reported in paediatric populations treated with asparaginase is that of cerebral thrombosis associated with hyperviscosity. ${ }^{7,8}$

The mechanism of L-asparaginase-associated hypertriglyceridaemia appears related to an increase in endogenous synthesis of very low density lipoprotein (VLDL) and/ or a decrease in the activity of lipoprotein lipase, a key enzyme in the catabolism of triglyceride-rich lipoproteins in the circulation. Corticosteroids can also increase hepatic VLDL secretion and thus may compound the effect. ${ }^{7,9}$ Finch et al. showed that while genetic risk factors were associated with baseline triglyceride levels in ALL, after treatment with L- or peg-asparaginase these genetic risk factors explained less of the variation in triglycerides, suggesting that hypertriglyceridaemia was driven by drug therapy more than genetics. ${ }^{11}$

The various different commercial presentations of therapeutic L-asparaginase have differed in their biological activity and thus their dosing regimens, despite being derived from native Escherichia coli strains. The development of the pegylated formulation has helped ameliorate some of the difficulties with administering the drug as well as reducing the tendency to antigenic reactions; ${ }^{12}$ however, it has made the monitoring for other toxicities even more important due to the prolonged action of the agent. A formulation derived from Erwinia bacteria is available for use in patients allergic to the $E$. coli formulations. It has no cross-reactivity, reduced immunogenicity and reportedly less coagulopathy. 
However, its cost is prohibitive in many areas and significant dose increases compared to the E. coli formulation have been required to achieve therapeutic drug levels; thus, further studies into its pharmacokinetics are required. ${ }^{1,12}$ Finch et al. found that the formulation impacted triglyceride levels, with peg-asparaginase associated with higher triglycerides compared to L-asparaginase. ${ }^{11}$

Interestingly, all four of our patients had T-cell malignancies. Studies involving much larger numbers of young adults with ALL/lymphoblastic lymphoma would be required to determine whether the lymphoid blast phenotype (B- or Tcell) has any causative role in the complications described. Pancreatitis, severe hyperlipidaemia and diabetes have all been reported as adverse events related to use of L-asparaginase, but are rarely problematic in children. Our experience suggests these toxicities may occur more frequently in the young adult population. Thus, we suggest consideration of baseline biochemical screening as well as serial monitoring of triglyceride, glucose and amylase/lipase in this population during L-asparaginase therapy, in addition to current routine blood tests. We would also advise remaining alert to the possibility of hyperlipidaemia first presenting as pseudohyponatraemia or other similar laboratory biochemical abnormalities that seem discordant with the clinical picture. Further review of large study populations may allow the identification of baseline markers to identify those young adults at increased risk of developing such toxicities, as well as clearly identifying the appropriate frequency of monitoring and thresholds for intervention.

Conflicts of interest and sources of funding: The authors state that there are no conflicts of interest to disclose.

\section{Jessica M. Heenan ${ }^{1}$, Amanda J. Hooper ${ }^{2,3}$, John R. Burnett ${ }^{2,3}$, Julian Cooney ${ }^{4}$}

${ }^{1}$ Department of Haematology, Launceston General Hospital, Launceston, Tas, Australia; ${ }^{2}$ Department of Clinical Biochemistry, PathWest Laboratory Medicine, Royal Perth Hospital and Fiona Stanley Hospital Network, Perth, WA, Australia; ${ }^{3}$ School of Medicine, University of Western Australia, Nedlands, WA, Australia; ${ }^{4}$ Department of Haematology, PathWest Laboratory Medicine, Fiona Stanley Hospital, Perth, WA, Australia

Contact Dr Julian Cooney.

E-mail: julian.cooney@ health.wa.gov.au

1. Shrivastava A, Khan AA, Khurshid M, et al. Recent developments in Lasparaginase discovery and its potential as anticancer agent. Crit Rev Oncol Hematol 2016; 100: 1-10.

2. Zhang J, Fan J, Venneti S, et al. Asparagine plays a critical role in regulating cellular adaptation to glutamine depletion. Mol Cell 2014; 56: 205-18.

3. Dolowy WC, Henson D, Cornet J, et al. Toxic and antineoplastic effects of L-asparaginase. Study of mice with lymphoma and normal monkeys and report on a child with leukemia. Cancer 1966; 19: 1813-9.

4. DeAngelo DJ, Stevenson KE, Dahlberg SE, et al. Long-term outcome of a pediatric-inspired regimen used for adults aged 18-50 years with newly diagnosed acute lymphoblastic leukemia. Leukemia 2015; 29: $526-34$.

5. Wang J, Ban MR, Zou GY, et al. Polygenic determinants of severe hypertriglyceridemia. Hum Mol Genet 2008; 17: 2894-9.

6. Siegel SE, Stock W, Johnson RH, et al. Pediatric-inspired treatment regimens for adolescents and young adults with Philadelphia chromosome-negative acute lymphoblastic leukemia: a review. JAMA Oncol 2018; 4: 725-34.
7. Cohen H, Bielorai B, Harats D, et al. Conservative treatment of L asparaginase-associated lipid abnormalities in children with acute lymphoblastic leukemia. Pediatr Blood Cancer 2010; 54: 703-6.

8. Lashkari HP, Lancaster D, Atra A, et al. Symptomatic severe hypertriglyceridaemia with asparaginase therapy in acute lymphoblastic leukaemia (ALL) and lymphoblastic lymphoma: is rechallenging safe? Int J Hematol 2011; 94: 571-5.

9. Parsons SK, Skapek SX, Neufeld EJ, et al. Asparaginase-associated lipid abnormalities in children with acute lymphoblastic leukemia. Blood 1997; 89: 1886-95.

10. Raja RA, Schmiegelow K, Sorensen DN, et al. Asparaginase-associated pancreatitis is not predicted by hypertriglyceridemia or pancreatic enzyme levels in children with acute lymphoblastic leukemia. Pediatr Blood Cancer 2017; 64: 32-8.

11. Finch ER, Smith CA, Yang W, et al. Asparaginase formulation impacts hypertriglyceridemia during therapy for acute lymphoblastic leukemia. Pediatr Blood Cancer 2020; 67: e28040.

12. Masurekar A, Fong C, Hussein A, et al. The optimal use of PEGasparaginase in relapsed ALL-lessons from the ALLR3 Clinical Trial. Blood Cancer J 2014; 4: e203.

DOI: https://doi.org/10.1016/j.pathol.2021.02.015

\section{Diagnostically challenging immunophenotypic shift in mantle cell lymphoma following ibrutinib and venetoclax therapy}

\section{Sir,}

A 57-year-old Caucasian male without a significant medical history presented with B symptoms, widespread lymphadenopathy and peripheral blood lymphocytosis. A diagnosis of mantle cell lymphoma (MCL) was confirmed on bone marrow histology and typical immunophenotype. He was treated with four cycles of R-HyperCVAD (rituximab, cyclophosphamide, vincristine, doxorubicin and dexamethasone) chemotherapy and obtained a complete metabolic remission on positron emission tomography (PET) scan. He then received maintenance rituximab 3 monthly (Fig. 1). One year later while on rituximab maintenance he had biopsy proven relapse of MCL in the right orbit and subcutaneously in the left posterior thigh. This was treated with local radiation to the thigh and orbit and he was commenced on ibrutinib. Six months later, he progressed with central nervous system (CNS) relapse of MCL. This was managed with intrathecal methotrexate and high dose cytarabine, followed by two further cycles of RHyperCVAD chemotherapy and craniospinal radiation, achieving a complete remission of the CNS disease. He was subsequently recommenced on ibrutinib maintenance. Three months later he presented with multiple skin lesions over the legs and torso, with a biopsy of both the left knee mass and skin lesions demonstrating blastoid MCL. He received palliative radiotherapy to the lower legs and proceeded to venetoclax monotherapy with achievement of complete response on PET. After 5 months of venetoclax therapy a bone marrow aspirate demonstrated no definite evidence of involvement with MCL; however, there were two focal areas of a lymphoid-appearing infiltrate which did not appear congruent with mantle cell lymphoma on immunohistochemistry (CD20, CD79a, PAX5, cyclinD1 negative). Flow cytometry did not demonstrate an aberrant or clonal B-cell population.

One month after this biopsy he presented with widespread bony pain, B symptoms, pancytopenia and a rapidly rising white cell count. Peripheral blood film demonstrated a 